\title{
Control of Transverse Motion for Quantum Gates on Individually Addressed Atomic Qubits
}

\author{
M. Cetina $\odot,{ }^{1,2, *}$ L.N. Egan $\odot,{ }^{1, \dagger}$ C. Noel $\odot,{ }^{1,2,3}$ M.L. Goldman $\odot,{ }^{1, \dagger}$ D. Biswas $\odot,{ }^{1}$ A.R. Risinger $\odot,{ }^{1}$ \\ D. Zhu, ${ }^{1, \dagger}$ and C. Monroe ${ }^{1,2,3,4}$ \\ ${ }^{1}$ Joint Quantum Institute, Center for Quantum Information and Computer Science, and Departments of Physics \\ and Electrical and Computer Engineering, University of Maryland, College Park, Maryland 20742, USA \\ ${ }^{2}$ Department of Physics, Duke University, Durham, North Carolina 27708, USA \\ ${ }^{3}$ Department of Electrical and Computer Engineering, Duke University, Durham, North Carolina 27708, USA \\ ${ }^{4}$ IonQ, Inc, 4505 Campus Drive, College Park, Maryland 20740, USA
}

(Received 13 July 2020; revised 15 January 2022; accepted 24 January 2022; published 2 March 2022)

\begin{abstract}
Individual trapped atomic qubits represent one of the most promising technologies to scale quantum computers, owing to their low idle errors and the ability to implement a full set of reconfigurable gate operations via focused optical fields. However, the fidelity of quantum gate operations can be limited by weak confinement of the atoms transverse to the laser. We present measurements of this effect by performing individually addressed entangling gates in chains of up to 25 trapped atomic ions. We present a semiclassical model that accurately describes the observed decoherence from the residual heating of the ions caused by noisy electric fields. We suppress these effects by cotrapping ancilla ions for sympathetically cooling the qubit ions throughout a quantum circuit.
\end{abstract}

DOI: 10.1103/PRXQuantum.3.010334

Isolated atomic qubits exhibit low idle decoherence and near-perfect replication. Individual atomic ions can be confined using electromagnetic fields and their qubits can be universally controlled with light $[1,2]$. Entangling quantum gates have been demonstrated between isolated pairs of trapped ions with fidelities exceeding $99.9 \%[3,4]$. In the work reported here, ion trap quantum computing systems have been extended to more than 20 qubits by trapping linear chains of ions, achieving entangling gate fidelity of $97.5 \%$. Further scaling of these systems to hundreds of qubits while increasing gate fidelities will likely require a modular architecture featuring the shuttling of ions through a multizone ion trap chip [5,6] or connecting modules through photonic interconnects [7]. In the presence of physical noise sources, the optimal number of ions in each zone remains an important open question.

In most trapped ion systems, entanglement between qubits in a chain is generated via a qubit-state-dependent optical force that drives normal modes of collective

*marko.cetina@duke.edu

${ }^{\dagger}$ Now at IonQ, Inc.

Published by the American Physical Society under the terms of the Creative Commons Attribution 4.0 International license. Further distribution of this work must maintain attribution to the author(s) and the published article's title, journal citation, and DOI. oscillations [8-10]. In a long ion chain, it is common to use high-frequency radial modes to mediate the entanglement [11], since these modes are more easily laser cooled to the ground state and are less susceptible to heating from electric field noise $[12,13]$. Control of the collective motion is accomplished via laser pulse shaping $[11,14]$. With individual optical addressing of each qubit, a single long chain of trapped ions allows for fully connected and reconfigurable quantum gate operations $[15,16]$ without any overhead required for shuttling.

In this work, we identify a limiting source of control noise relating to the individual optical addressing of large chains of atomic ion qubits. Here, the ions' motion in the weakly confined axial direction can spoil their coupling to the tightly focused individual-addressing laser beams [17]. A similar effect may also limit gate fidelities in arrays of individually addressed neutral atoms [18].

The effect of axial motion is magnified as the ion chain gets longer and its susceptible long-wavelength modes are easily heated by noisy electric fields. We present a model of the induced decoherence with supporting measurements in a leading trapped ion system based on a single long chain [19]. In this system, the heating effects are well explained by the motion in the lowest-frequency axial mode. We calculate that, in near-equispaced chains, the frequency of this mode is approximately inversely proportional to the ion number. Since the electric spectral density of electric field noise in ion traps typically follows a power 
law, the resulting addressing errors increase sharply with system size and run time.

Finally, we show that this noise source can be suppressed by sympathetically cooling the qubits [20,21], and we do so by employing a novel cooling scheme that uses ${ }^{172} \mathrm{Yb}^{+}$. We show measurements that confirm the scheme will not be limited by fundamental atomic processes, and faces only control challenges in the implementation.

This article is organized as follows. In Sec. I we present a semiclassical model of the effects of axial motion of an ion chain. In Sec. II A, we confirm this model by measurements with a single trapped ion. In Sec. II B, we consider the effects of heating the ion chains by background electric fields and, in Sec. II C, we consider the impact of this heating on entangling gates. Finally, in Sec. III, we demonstrate a sympathetic cooling scheme to counter this heating effect and characterize the qubit decoherence induced by this cooling.

\section{EFFECT OF AXIAL MOTION ON ION ADDRESSING: SEMICLASSICAL MODEL}

We consider the effect of axial $(\hat{x})$ motion of a chain of trapped ions, each of mass $M$, with axial normal mode frequencies $\omega_{m}$. The ions are addressed by an array of focused laser beams that drive Rabi oscillations between two qubit states $(|0\rangle$ and $|1\rangle)$. The beams are directed perpendicular to $\hat{x}$ so that, throughout their axial motion, the ions experience fixed phases of the Rabi drive. The instantaneous qubit Rabi frequency $\Omega_{i}$ of the ion $i$ is proportional to the electric field amplitude of the laser beam at the position of this ion [1,14]. This is true for both direct optical qubit transitions [1] or two-beam optical Raman transitions where one of the beams has a uniform intensity profile, as used here [14].

To second order in the position $x_{i}$ of the $i$ th ion relative to its equilibrium, the instantaneous Rabi frequency of this ion can be written as $\Omega_{i}=\Omega_{i, 0}+\Omega_{i, 0}^{\prime} x_{i}+\Omega_{i, 0}^{\prime \prime} x_{i}^{2} / 2$, where $\Omega_{i, 0}$ is the Rabi frequency at the equilibrium position of ion $i$ and $\Omega_{i, 0}^{\prime \prime}$ is its curvature along the $\hat{x}$ axis.

The operator $\hat{x}_{i}$ corresponding to the position $x_{i}$ can be decomposed into axial normal modes of the ion chain as

$$
\hat{x}_{i}=\sum_{m=0}^{N-1} b_{i m} \sqrt{\frac{\hbar}{2 M \omega_{m}}}\left(\hat{a}_{m}^{\dagger}+\hat{a}_{m}\right),
$$

where $b_{i m}$ is the participation of this ion in axial mode $m\left(\sum_{m} b_{i m} b_{j m}=\delta_{i j}\right)$ [22]. Our amplitude-modulated twoqubit gates are designed to ensure that no information is left behind in the ions' motion after the gate. To achieve this, the amplitude profiles of the two-qubit gate waveforms are smooth over time $t_{g}=1 / \Delta_{r}$, where $\Delta_{r}<2 \pi \times$ $15 \mathrm{kHz}$ is the spacing between the neighboring radial modes. Since $\Delta_{r}$ is much smaller than all the axial mode frequencies $\omega_{m}$, in two-qubit gates, $\Omega_{i}$ changes slowly on the scale of the axial motion. Therefore, we may replace $\hat{x}_{i}$ by its average value of 0 , and $\hat{x}_{i}^{2}$ by its average value of $\sum_{m} b_{i m}^{2} E_{m} /\left(M \omega_{m}^{2}\right)$, where $E_{m}=\hbar \omega_{m}\left(n_{m}+1 / 2\right)$ is the energy of excitation of mode $m$, with $n_{m}$ the number of motional quanta in the same mode. Taking the spatial spread of each ion's wavepacket to be much smaller than the size of its laser beam and $\Omega_{i} \ll \omega_{m}$, ion $i$ will then experience a time-averaged Rabi frequency

$$
\bar{\Omega}_{i}=\Omega_{i, 0}+\frac{1}{2} \Omega_{i, 0}^{\prime \prime} \sum_{m=0}^{N-1} b_{i m}^{2} \frac{E_{m}}{M \omega_{m}^{2}} .
$$

The leading effect of the fluctuations in $x_{i}$ takes the form of a state-dependent axial force proportional to the gradient of the Rabi frequency along the axial direction [23]. This force can be suppressed by centering the ions on the addressing beams and by employing pulse shaping [11] to control their coupling to axial modes.

If qubit $i$ starts in $|0\rangle$ and is driven resonantly, the probability of finding the qubit in $|1\rangle$ can be evaluated by performing the thermal average of the Rabi oscillation over all the modes $m$ as

$$
p_{|1\rangle}(t)=\prod_{m=0}^{N-1} \sum_{n_{m}=0}^{\infty} p_{m}\left(n_{m}\right) \sin ^{2}\left(\frac{\bar{\Omega}_{i} t}{2}\right),
$$

where $p_{m}\left(n_{m}\right)$ are the occupation numbers of mode $m$.

We assume that the energies $E_{m}$ follow a thermal Boltzmann distribution at temperatures $T_{m}$, leading to a wide distribution of the mean Rabi frequencies $\bar{\Omega}_{i}$ [17]. In the regime that is discussed in the main text, the mean occupation numbers $\bar{n}_{m} \approx k_{B} T_{m} /\left(\hbar \omega_{m}\right)$ of the modes that contribute to the gate error are much larger than one. Expanding Eq. (3) to leading order in $\hbar \omega_{m} / k_{B} T_{m} \sim 1 / n_{m}$, we obtain

$$
p_{|1\rangle}(t)=\frac{1-C \cos \left(\Omega_{i, 0} t+\phi\right)}{2} .
$$

The Rabi oscillations exhibit a phase advance of $\phi=$ $\sum_{m} \arctan \left(\Omega_{i, 0} \theta_{i m} t\right)$ and a loss in contrast by a factor of $C=\prod_{m}\left(1+\theta_{i m}^{2} \Omega_{i, 0}^{2} t^{2}\right)^{-1 / 2}$. Here, the decay parameter for ion $i$ due to mode $m$ is defined as

$$
\theta_{i m}=-\frac{k_{B} T_{m} b_{i m}^{2}}{2 M \omega_{m}^{2}} \frac{\Omega_{i, 0}^{\prime \prime}}{\Omega_{i, 0}}=-b_{i m}^{2} \xi_{m}^{2} \frac{\Omega_{i, 0}^{\prime \prime}}{\Omega_{i, 0}} \bar{n}_{m}
$$

and characterizes the decoherence per Rabi cycle of ion $i$ due to the motion in mode $m$. Here, $\xi_{m}=\sqrt{\hbar /\left(2 M \omega_{m}\right)}$ is the zero-point spatial spread of the wavefunction of a single ion in a trap with frequency $\omega_{m}$ (for an ${ }^{171} \mathrm{Yb}^{+}$ion with $\omega_{0}=2 \pi \times 100 \mathrm{kHz}, \xi_{0}=17 \mathrm{~nm}$ ). 


\section{EFFECTS OF AXIAL MOTION ON ATOMIC QUBITS}

\section{A. Axial heating of a single ion}

To probe the decoherence caused by the thermal axial motion of the qubit, we confine a single ${ }^{171} \mathrm{Yb}^{+}$ion in a microfabricated surface-electrode linear $\mathrm{rf}$ ion trap (HOA2.1.1 by the Sandia National Laboratories [24]), obtaining radial secular frequencies of about $3 \mathrm{MHz}$. We address this ion with a pair of pulsed 355-nm laser beams that drive the $F=0(|0\rangle) \rightarrow F=1, m_{F}=0(|1\rangle)$ ground-state hyperfine transition via a Raman process. The Raman beams are perpendicular both to each other and to the trap axis $\hat{x}$. One of the two beams is tightly focused while the other is more than 10 times larger. Following Raman sideband cooling [25] of the radial motion of the ion to an average occupation number $\bar{n}$ of less than 0.15 quanta, we optically pump the ion into $|0\rangle$. After a 5-ms delay, we use the Raman beams to drive carrier Rabi oscillations of the ion, obtaining the data shown in Fig. 1. We observe that, when the axial frequency of the ion is decreased from 700 to 140 $\mathrm{kHz}$, the Rabi oscillations exhibit a sharp decay [17]. The observed oscillations do not exhibit an exponential decay with a constant phase shift, as would be expected from pure phase damping [26], but instead agree with the model from Eqs. (4)-(5).

We investigate the spatial dependence of the decoherence by applying static trap voltages to move the ion along $\hat{x}$. First, we map out the spatial profile of our tightly focused Raman beam by tightly confining the ion and

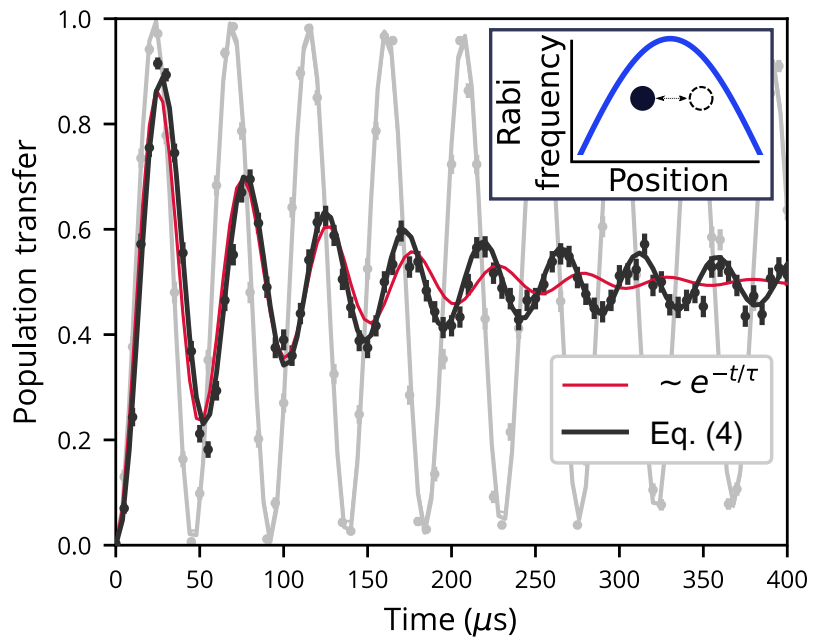

FIG. 1. Rabi oscillations between the ground hyperfine clock states of a single trapped ${ }^{171} \mathrm{Yb}^{+}$ion with axial frequencies 140 $\mathrm{kHz}$ (black) and $710 \mathrm{kHz}$ (gray). The ion is prepared in the state $|0\rangle$ and driven by a pair of 355-nm Raman beams. The black and gray lines correspond to fits to Eq. (4). The red line corresponds to a fit to a two-state model with phase damping. Inset: illustration of the axial motion of the ion in the tightly focused Raman beam. then driving it with a carrier Raman pulse of duration $\tau=0.5 \mu \mathrm{s}$. We use the fraction of the ion population transferred to $|1\rangle$ to determine the Rabi angle $\Omega_{1,0} \tau$, as shown in Fig. 2(a). A Gaussian fit to the obtained data $\left(\Omega_{1,0} \tau \sim e^{-x^{2} / w^{2}}\right)$ yields the $1 / e^{2}$ intensity radius of the tightly focused beam $w=870(25) \mathrm{nm}$. On one shoulder of the tightly focused beam, we observe a deviation from the Gaussian shape, which we ascribe to the mode profile of the laser.

Next, we relax the single ion's axial confinement and perform a carrier Rabi oscillation experiment, as in Fig. 1, at each set position of the ion. We fit the resulting data to the model from Eq. (4) to extract the decay parameter $\theta \equiv \theta_{10}$, with the results shown in Fig. 2(b). We compare the obtained $\theta(x)$ data to the prediction $\theta=2\left(\xi_{0} / w\right)^{2}(1-$ $\left.2 x^{2} / w^{2}\right) \bar{n}_{0}$, which assumes a Gaussian shape of the tightly focused beam, with the ion's axial average thermal vibrational number of $\bar{n}_{0}=280$ at the expected Doppler cooling temperature. We observe good agreement on the left shoulder of the individual-addressing beam. We ascribe the increased value of $\theta$ on the right shoulder to the deviation of the laser's mode shape from a Gaussian, which is evident in Fig. 2(a). In striking contrast to decoherence caused by beam pointing fluctuations, which increase on the sides of the focused beam, the measured decay parameter reaches its minimum values near the inflection points of the Gaussian curve, indicated by the vertical dashed lines in Fig. 1. The induced decay measurements from Figs. 1
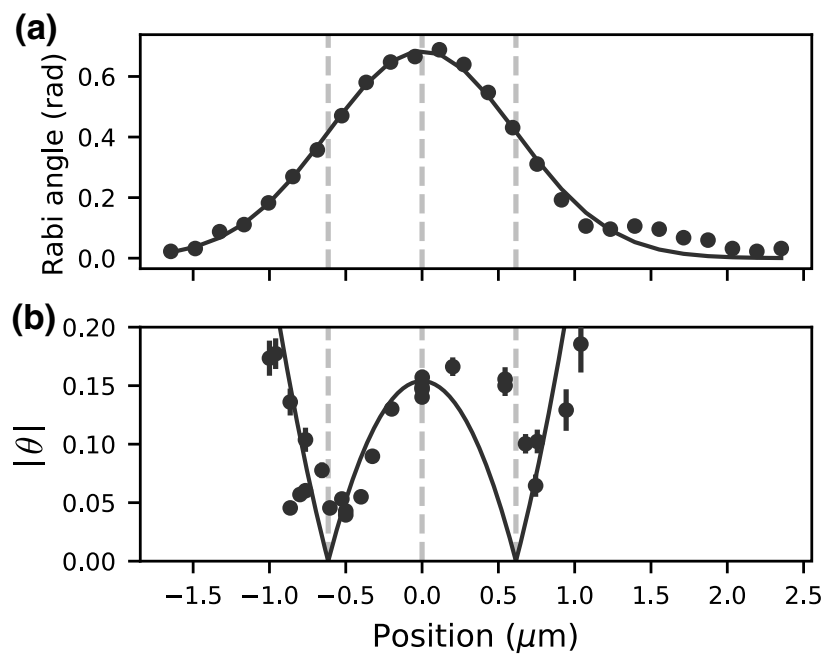

FIG. 2. (a) The angle of the Rabi oscillation of a single ${ }^{171} \mathrm{Yb}^{+}$ ion driven by a $5-\mu$ s Raman pulse depending on the position of the ion in a trap with tight axial confinement $\left(\omega_{0}=2 \pi \times 630\right.$ $\mathrm{kHz}$ ). (b) The absolute value of the decay parameter obtained by fit of the Rabi oscillation to the model from Eq. (4), for a weakly axially confined ion $\left(\omega_{0}=2 \pi \times 140 \mathrm{kHz}\right)$ depending on the position of the ion. The solid line indicates a prediction based on the Gaussian fit from Fig. 2(a) and an axial ion temperature corresponding to $\bar{n}=280$ quanta. 
and 2 demonstrate that both the spatial and the temporal behavior of the observed Rabi oscillations of an ion under weak axial confinement are well described by the model of Eqs. (4)-(5).

\section{B. Axial heating of a chain of ions}

We now consider the effect of axial motion on an array of atomic ions. After sufficient time $t_{w}$ without laser cooling, the temperature $T_{m}$ of the axial mode $m$ will be dominated by the work done by noisy background electric fields $[12,13]$ during time $t_{w}$. If this field is uniform in space, its work on mode $m$ will be proportional to $\left(\sum_{i} b_{i, m}\right)^{2}$. Since the contribution of the $m$ th mode to the decay parameter scales as $\omega_{m}^{-2}$, and, for higher-frequency modes, $b_{i, m}$ oscillates with the ion index $i$ (i.e., neighboring ions move out of phase with each other), we expect the heating of the lowest-frequency "in-phase" axial mode $(m=0)$ to strongly dominate the gate error budget. In this case, the effects of the axial motion on the $i$ th ion are captured by the single decay parameter

$$
\theta_{i} \equiv \theta_{i 0}=b_{i 0}^{2}\left(\sum_{j} b_{j 0}\right)^{2} \theta\left(t_{w}\right),
$$

where $\theta\left(t_{w}\right)$ is the decay parameter of a single ion in a trap with axial frequency equal to $\omega_{0}$, following Eq. (5). Note that, for harmonic axial confinement where the in-phase mode is the center-of-mass mode $\left(b_{i 0}=N^{-1 / 2}\right)$, the decay parameter $\theta_{i}$ becomes equal to that for a single ion.

In the experiment, we use a combination of quadratic and quartic axial potentials [27] to prepare near-equispaced chains of $15(25){ }^{171} \mathrm{Yb}^{+}$ions with $4.4 \mu \mathrm{m}$ ion spacing, obtaining $193(123) \mathrm{kHz}$ as the lowest axial mode frequency. We use a 32-channel acousto-optic modulator [14] to produce individually controlled, tightly focused $355-\mathrm{nm}$ beams identical to that from Fig. 2. Following sideband cooling of the radial modes of the ion chain, we drive simultaneous Raman Rabi oscillations on the middle 13 (15) ions in the chain after a variable wait time $t_{w}$ following the Doppler and sideband cooling. We fit the oscillations of each ion to the model from Eqs. (4)-(5) to determine the rate of change of its decay parameter, and we show the results of these measurements in Fig. 3. The observed variation of the decay parameters across the chains follows the factor $b_{i 0}^{2}$ from Eq. (6), with the Rabi oscillations of the middle ions exhibiting increased decay due their higher participation in the lowest-frequency axial mode.

Electric field noise in ion traps is empirically observed to follow a power law scaling with frequency $\omega^{-\alpha}$, with exponent $\alpha$ between 0 and 2 [13]. To check for consistency of our observations with this behavior, we use sideband spectroscopy of a single ion to measure the heating rate of $\dot{\bar{n}}_{r}=88(6)$ quanta/s for a $3-\mathrm{MHz}$ radial mode parallel to the trap surface. In Fig. 3, we show the predictions based

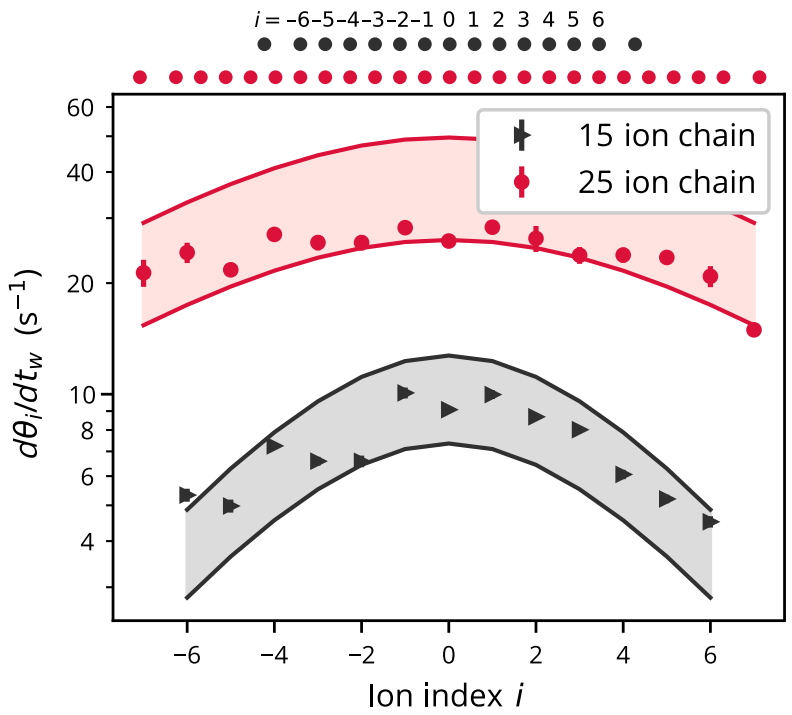

FIG. 3. The rate of change of the decay parameter $\theta_{i}$ as a function of the ion index $(i)$ in near-equispaced chains of 15 ions (black) and 25 ions (red). The error bars are statistical from fits of $\theta_{i}\left(t_{w}\right)$ to a linear increase with the wait time $t_{w}$. The solid lines correspond to predictions based on the power-law spectral density of electric field noise and the independently measured heating rate of one ion at $3 \mathrm{MHz}$ of $\dot{\bar{n}}_{r}=88(6)$ quanta/s. For $\alpha=0.8$, we obtain $6 \times 10^{5}\left(1.7 \times 10^{5}\right)$ quanta/s for the heating rate of the lowest axial mode of 25 (15) ions while, for $\alpha=1$, we obtain $1.2 \times 10^{6}\left(3 \times 10^{5}\right)$ quanta/s.

on Eqs. (5) and (6) and $\alpha=1$. We also show predictions for $\alpha=0.8$, which we deduced independently from $d \theta / d t_{w}$ for a single ion as a function of the axial trap frequency (see Appendix A). We observe good quantitative agreement with our data, suggesting that electric field noise in our system is consistent with previously observed values.

\section{Effect on entangling gates}

The Rabi frequency $\Omega_{i, j}$ of the entangling dynamics between the $|00\rangle$ and $|11\rangle$ states of ions $i$ and $j$ during twoqubit entangling gates [8-10] is proportional to the product of the single-qubit Rabi frequencies on the two addressed ions. If the two ions are centered on the maxima of their respective individual-addressing beams, the joint decay parameter corresponding to axial mode $m$ is $\theta_{i m}+\theta_{j m}$. The fidelity of the obtained two-qubit state is bounded from above by $F_{i j}=\operatorname{Tr}\left[\hat{\rho}(t) \hat{\rho}_{\chi}\right]=\left\langle\cos ^{2}\left(\bar{\Omega}_{i, j} t-\chi\right)\right\rangle$, where $\chi$ is the desired two-qubit gate angle [8] and $\bar{\Omega}_{i, j}$ is the mean value of $\Omega_{i, j}$ during the ions' axial motion. Performing the thermal average as in Eq. (4), we find that the state fidelity bound after $N_{g}$ successive fully entangling gates $\left(\chi=N_{g} \pi / 4\right)$ is

$$
F_{i j}=\frac{1}{2}+\frac{1}{2} \prod_{m} \frac{1}{\sqrt{1+\left(N_{g} \pi / 2\right)^{2}\left(\theta_{i m}+\theta_{j m}\right)^{2}}} .
$$


To check this prediction, we apply amplitude modulation to the focused Raman beams addressing two particular ions in the above chains of 15 (25) ions so as to perform one or three successive 250- (500-) $\mu$ s-long entangling gates between the target ions [14], after a variable wait time $t_{w}$ following Doppler and sideband cooling. After applying the entangling gate(s), we measure the $\{|00\rangle,|11\rangle\}$ subspace population $p_{00}+p_{11}$. Separately, we apply additional $\pi / 2$ pulses with variable phase to both ions and extract the parity fringe contrast $C$, to witness entanglement [28]. We compensate for the reduced mean laser power due to axial heating by adjusting the laser power to maximize the gate fidelity. We independently determine $\theta_{i}$ and $\theta_{j}$ in our chains by repeating the measurements from Fig. 3 and fitting the resulting decay parameters to a linear increase with the wait time $t_{w}$.

We compute the gate fidelities from our measurements as $F=\left(p_{00}+p_{11}+C\right) / 2[28]$ and show these results in Fig. 4. With 15 ions, we achieve a baseline two-qubit gate fidelity of $98.5 \%$ and with 25 ions we achieve a gate fidelity of $97.5 \%$ on the two selected ions. We compare these results to the predictions based on Eq. (7) using the previously measured $\theta$ values corresponding to the middle of the gate sequence. We observe good agreement between our measurements and the model of Eq. (7). In particular, in chains of 15 (25) ions, after $t_{w}=10(2.5) \mathrm{ms}$, corresponding to the time it takes to complete 40 (5) sequential

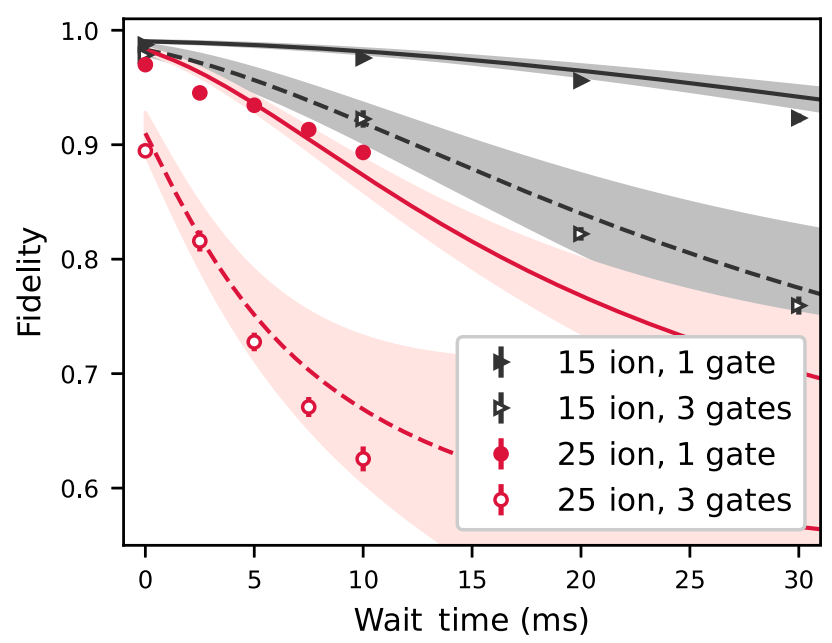

FIG. 4. The fidelity of one (three) fully entangling gate(s) on two ions (indices $i=-6$ and $j=-5$ as in Fig. 3) in a chain of 15 and 25 ions as a function of the waiting time before the gate(s). The error bars denote $1 \sigma$ uncertainty of the weighted average of several measurements of the fidelity. The shaded areas show the predictions based on Eq. (7) using the average axial temperature during the gate sequence, adjusted down by the error in state preparation and measurement in our ion chains $(0.9 \%$; see Appendix C). The uncertainty in the theory predictions reflects the errors in our determination of the average decay parameters $\theta_{i}$ and $\theta_{j}$. individual entangling gates, our model explains most of the observed loss in gate fidelity.

In harmonic ion chains, $\omega_{0}$ is roughly inversely proportional to the ion number $N$ [29]. As shown in Appendix B, this remains true in the near-equispaced chains that are considered here. Assuming electric field noise with exponent $\alpha$ and using Eqs. (5)-(6), we obtain $d \theta_{i} / d t_{w} \sim N^{2+\alpha}$ in such chains, implying that the entangling gate error scales as $t_{w}^{2} N^{4+2 \alpha}$. For $\alpha \approx 1$, as seen in many ion trap experiments [13], this results in a scaling of gate errors proportional to $N^{6}$, which would limit the use of long chains with high-fidelity operations.

\section{AXIAL SYMPATHETIC COOLING WITH ${ }^{172} \mathbf{Y b}^{+}$}

Axial heating of the ion chain can be countered by interspersing the coherent operations with periodic sympathetic cooling [20] of axial modes via coolant ions that are distributed throughout the ion chain $[6,20,21,27]$. Since the gate error is proportional to $t_{w}^{2}$, in the limit of frequent cooling to the same initial axial temperature $T_{0}$, we expect sympathetic cooling to strongly suppress the effects of axial heating on gate fidelity.

We explore sympathetic cooling by cotrapping ten ${ }^{171} \mathrm{Yb}^{+}$and five ${ }^{172} \mathrm{Yb}^{+}$ions in the trap from Figs. 3 and 4.

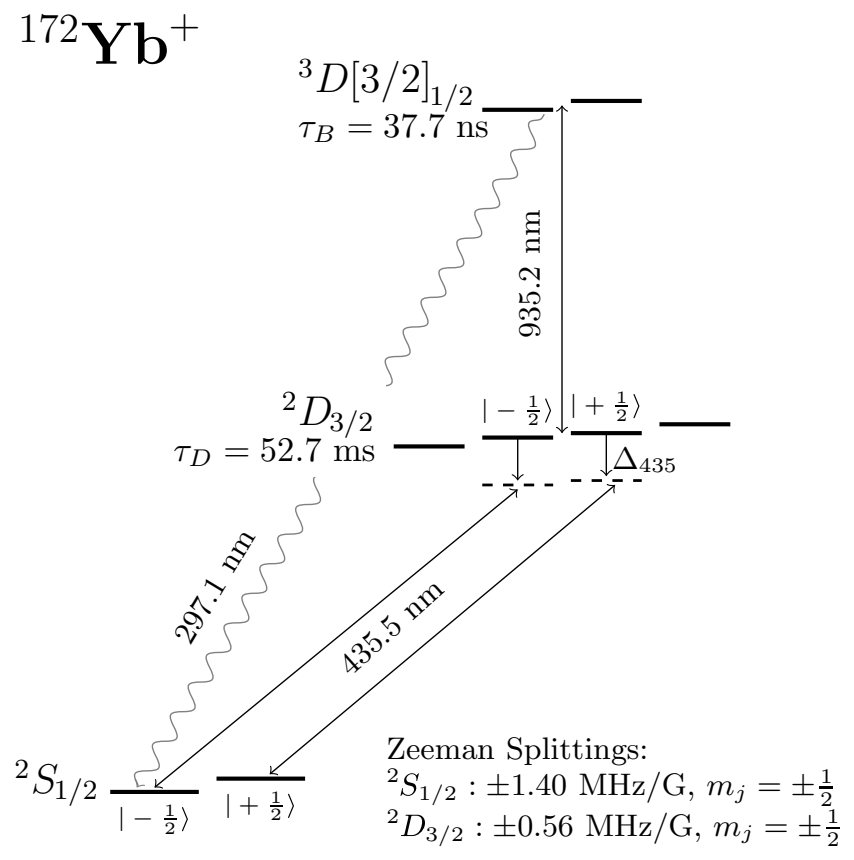

FIG. 5. Diagram of the states and transitions involved in the cooling of ${ }^{172} \mathrm{Yb}^{+}$, based on data from Refs. [30-32]. Motional energy is removed by simultaneously addressing the $\Delta m=0$, ${ }^{2} S_{1 / 2}{ }^{2} D_{3 / 2}$ quadrupole transitions with the same detuning $\Delta_{435}$. State reset is performed by excitation using a 935-nm laser, followed by emission of a $297-\mathrm{nm}$ photon. 
We adapt the ${ }^{171} \mathrm{Yb}^{+}$narrow-line sideband cooling scheme from Ref. [33] to the ${ }^{172} \mathrm{Yb}^{+}$ions (Fig. 5). To cool the axial motion of the ion chain, we direct a $18-\mu \mathrm{m}$-waist, $20-\mathrm{mW}$, $435-\mathrm{nm}$ laser beam parallel to the trap surface and at $45^{\circ}$ to the chain axis, and detune it by $\Delta_{435}=-2 \pi \times 500 \mathrm{kHz}$ relative to the ${ }^{2} S_{1 / 2}-{ }^{2} D_{3 / 2}$ quadrupole transition in ${ }^{172} \mathrm{Yb}^{+}$ (Fig. 5). We maximize the coupling of this beam to both ground Zeeman states in ${ }^{172} \mathrm{Yb}^{+}$by using a linear polarization perpendicular to the trap surface, applying a magnetic field of $3.2 \mathrm{G}$ nearly parallel to the $x$ axis and using two equal $435-\mathrm{nm}$ tones separated by the $5.3-\mathrm{MHz}$ Zeeman splitting to drive both $\Delta m=0$ transitions, each with peak carrier Rabi frequency $\Omega_{435}$. The state of the ${ }^{172} \mathrm{Yb}^{+}$ions is reset by spontaneous emission of a 297-nm photon following continuous resonant excitation of the ${ }^{2} D_{3 / 2}{ }^{3} D[3 / 2]_{1 / 2}$ transition at the rate $\Gamma_{935}=2.5 \times 10^{6} / \mathrm{s}$, determined by a measurement of the broadening of the ${ }^{2} D_{3 / 2}{ }^{3} D[3 / 2]_{1 / 2}$ line (Fig. 5).

We probe the axial temperature of the ion chain by driving carrier Rabi oscillations of the middle $\left({ }^{171} \mathrm{Yb}^{+}\right)$ion and fitting these to Eq. (4) to extract the decay parameter $\theta$ [Fig. 6(a)]. In a pure ${ }^{171} \mathrm{Yb}^{+}$chain, over the course of a 15-ms wait following sideband cooling, the fitted decay parameter for the middle ion increases from $\theta=$ 0.041(3) [gray curve in Fig. 6(a)] to 0.194(8) [black curve in Fig. 6(a)]. Replacing five ${ }^{171} \mathrm{Yb}^{+}$ions with ${ }^{172} \mathrm{Yb}^{+}$and performing $3 \mathrm{~ms}$ of sympathetic cooling after the wait lowers the fitted decay parameter to 0.029(3) [blue curve in Fig. 6(a)], indicating that the effect of axial heating is reversed by the applied cooling.

We characterize the cooling process by recording Rabi oscillations following a $15-\mathrm{ms}$ wait for different $435-\mathrm{nm}$ laser intensities and cooling times [Fig. 6(b)]. The data shown correspond to an average over random orderings of the coolant and data ions. For $\Omega_{435} /(2 \pi)=640 \mathrm{kHz}$, we obtain a cooling time of $\tau=0.91(2) \mathrm{ms}$, with equilibrium axial temperature corresponding to $\langle\theta\rangle=0.037(2)$.

To find the minimal residual XX errors due to axial motion, we consider a $50 \%$ cooling duty cycle. In this setup, the exponential fit to the data from Fig. 6 for $\Omega_{435}=$ $2 \pi \times 640 \mathrm{kHz}$, together with the mean heating from Fig. 3 predicts $\langle\theta\rangle=0.045$ during the XX gates, corresponding to $5 \times 10^{-3}$ mean error due to axial motion for a single fully entangling two-qubit XX gate in a chain of 15 ions. We expect that optimizing the 935-nm and 435-nm detunings and powers, and adding radial $435-\mathrm{nm}$ cooling (see Appendix D), can reduce the equilibrium value of $\langle\theta\rangle$ to 0.02 and thereby bring the residual XX error in a 15 -ion chain close to the best entangling gates in isolated pairs of trapped ions $[3,4]$.

The sympathetic cooling process can induce decoherence of the ${ }^{171} \mathrm{Yb}^{+}$qubits by direct scattering of 435 $\mathrm{nm}$ photons, as well as by off-resonant absorption of the spontaneously emitted photons on the ${ }^{3} D[3 / 2]_{1 / 2}{ }^{2} S_{1 / 2}$ transition $(\lambda=297 \mathrm{~nm})$. We measure the decoherence of our qubits using a microwave Carr-Purcell-Meiboom-Gill sequence [34] (Fig. 7). We sweep the phase of the last $\pi / 2$ pulse and record the average contrast of the resulting fringe across the ten ${ }^{171} \mathrm{Yb}^{+}$ions depending on the total cooling time with $\Omega_{435}=2 \pi \times 640 \mathrm{kHz}$ (blue points in Fig. 7). A linear fit indicates that the obtained contrast decreases at the rate of $4(1) \times 10^{-2} / \mathrm{s}$ or $1.2(3) \times 10^{-4}$ per qubit, per one 3-ms-long cooling cycle, corresponding to an error of at most $6(2) \times 10^{-5}$ per qubit, per 3-ms-long cooling
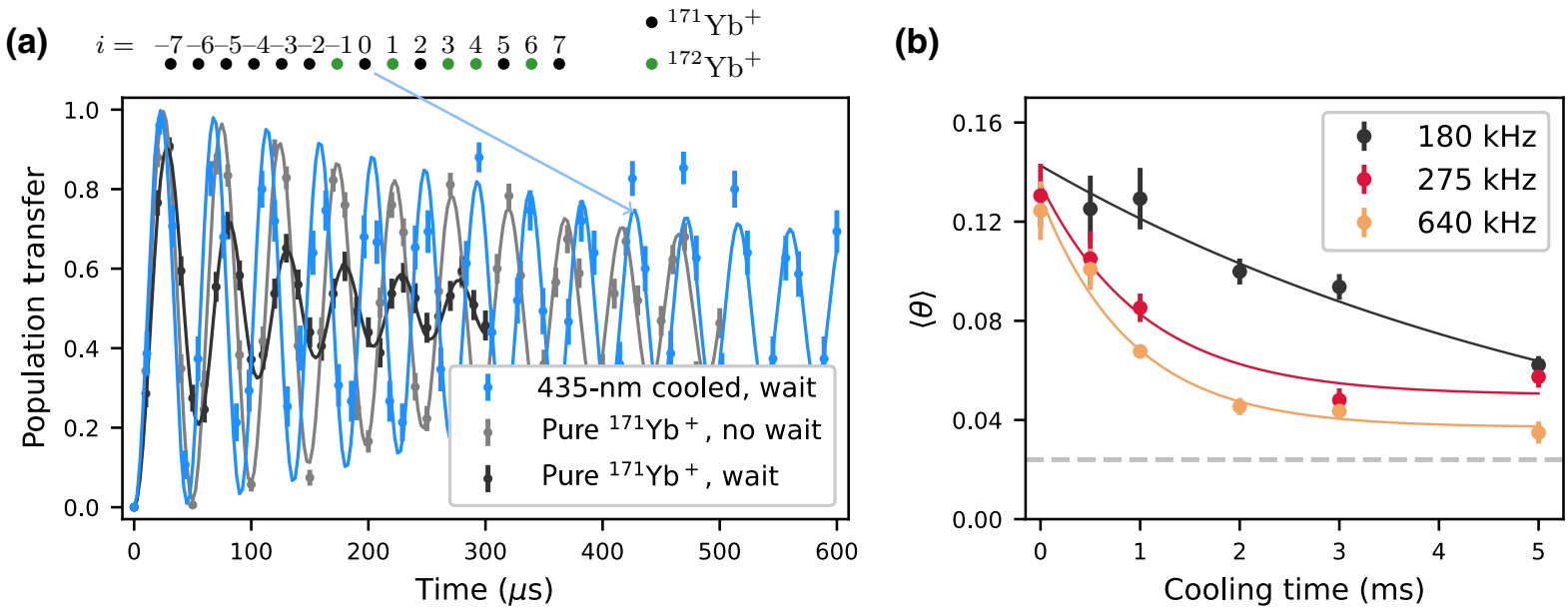

FIG. 6. (a) Carrier Rabi oscillations of the middle ${ }^{171} \mathrm{Yb}^{+}$ion $(i=0)$ in a mixed-species chain after a 15 -ms wait followed by 3 ms of sympathetic cooling with $\Omega_{435}=2 \pi \times 275 \mathrm{kHz}$ (blue), compared to a single-species chain after a 0 -ms wait (gray), and a 15-ms wait (black). The data are fitted to Eq. (4). (b) Mean decay parameter of Rabi oscillations $\langle\theta\rangle$ of the ten ${ }^{171} \mathrm{Yb}^{+}$ions after sympathetic cooling, as a function of the cooling time for $\Omega_{435}=2 \pi \times 180 \mathrm{kHz}$ (black), $275 \mathrm{kHz}$ (red), $640 \mathrm{kHz}$ (yellow). The experimental data are fitted to exponential decays with offsets (thin lines). The mean decay parameter across the chain $\langle\theta\rangle=0.024$ for a pure ${ }^{171} \mathrm{Yb}^{+}$ chain following sideband cooling is indicated by the gray dashed line. 


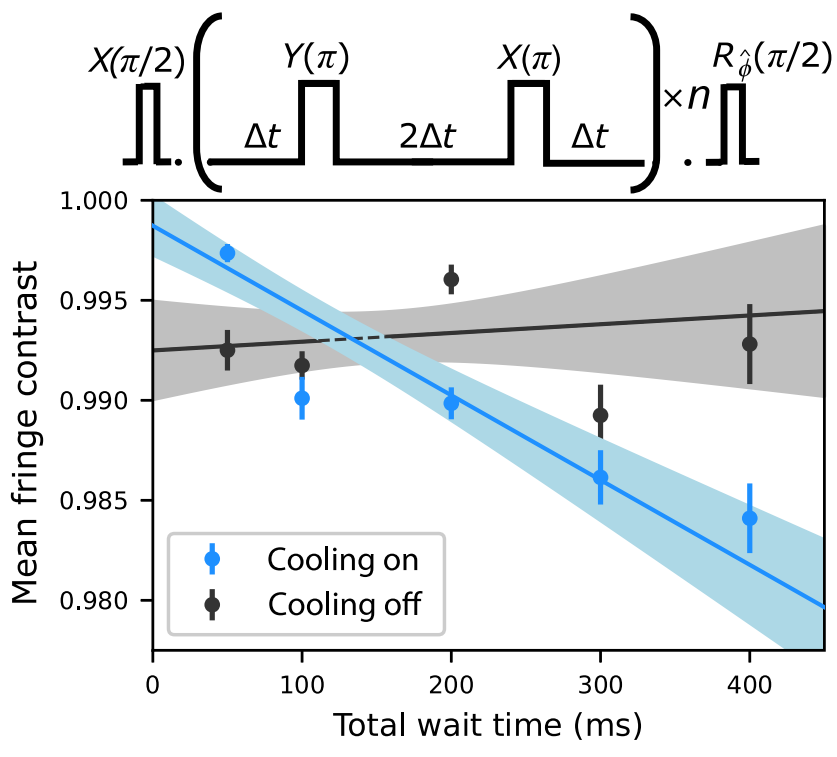

FIG. 7. The mean fringe contrast of the ${ }^{171} \mathrm{Yb}^{+}$qubits in a chain of ten ${ }^{171} \mathrm{Yb}^{+}$and five ${ }^{172} \mathrm{Yb}^{+}$ions after a CPMG microwave pulse sequence as a function of the total sequence wait time $4 n \Delta t$, where $\Delta t=6.25 \mathrm{~ms}$ (see the top schematic), with 435-nm cooling on (blue points) and off (black points) during the wait times. The fitted lines have slopes of $-0.04(1) / \mathrm{s}$ (blue) and $0.004(15) / \mathrm{s}$ (black). The shaded areas correspond to $1 \sigma$ confidence intervals of the fits.

cycle. The observed decoherence rate is about 7 times higher than the predicted decoherence, which is dominated by Rayleigh and Raman scattering of the 435-nm photons on the D1 and D2 transitions (see Appendix E). To explain the additional decoherence, we simulated the effect of the fluctuations of the Stark shift caused by the 435$\mathrm{nm}$ light [220(40) Hz measured mean shift] on our CPMG sequence. Using the measured power fluctuations of our 435-nm laser, corresponding to $1 \% \mathrm{rms}$ fractional noise in the laser's power, we obtain good agreement with our data, indicating that power stabilization of the 435-nm light can remove our dominant source of decoherence during cooling.

\section{CONCLUSION AND OUTLOOK}

In this work, we identify a process driven by electric field noise in Paul traps that limits the fidelity of individually addressed gates in long ion chains. We find that the error of entangling gates due to this process is expected to scale as $t_{w}^{2} N^{6}$, where $N$ is the number of ions and $t_{w}$ the wait time since cooling.

We show in Sec. III that, in a medium-length chain, this process can be effectively countered with sympathetic cooling between gates. By using ${ }^{172} \mathrm{Yb}^{+}$as a coolant for ${ }^{171} \mathrm{Yb}^{+}$in a novel cooling scheme, in a 15 -ion chain we are able to reverse decoherence caused by a $t_{w}=15$ ms wait time (about 60 entangling gates) by sympathetically cooling for $3 \mathrm{~ms}$. The order of multispecies ions in our experiments is random; future work on reordering ions in a chain should allow us to explore optimal sympathetic cooling under different permutations and combinations of ${ }^{171} \mathrm{Yb}^{+}$and ${ }^{172} \mathrm{Yb}^{+}$ions. Optimal cooling solutions will likely favor ${ }^{172} \mathrm{Yb}^{+}$coolant ions towards the center of the chain $(i=0)$, where their participation in the lowest-frequency axial mode is highest.

As the axial confinement decreases with chain length, long chains will require additional cooling power per ion to counter the heating due to electric field noise, potentially decreasing the duty cycle of the system. Even this can be countered by axially compressing the ion chain to stiffen it, surface cleaning, and cryogenic operation of ion traps, likely allowing individually addressed entangling gates in chains of more than 50 ions. Confinement of the whole chain in an optical lattice [35] or of select ions in optical tweezers [36] may further increase this number to hundreds of ions.

\section{ACKNOWLEDGMENTS}

This work was performed at the University of Maryland (UMD) using cleanroom facilities of the UMD FabLab, with no laboratory or equipment support from IonQ. We acknowledge fruitful discussions with M. Revelle, V. Vuletić, J. Kim, M. Li, N. Pisenti, and K. Wright; and the contributions of J. Amini, K. Beck, K. Hudek, and J. Mizrahi to the experimental setup. We thank the anonymous referee for pointing out the leading theoretical source of qubit decoherence in our cooling scheme. This work is supported by the ARO through the IARPA LogiQ program, the NSF STAQ Program, the AFOSR MURIs on Dissipation Engineering in Open Quantum Systems and Quantum Measurement/Verification and Quantum Interactive Protocols, the ARO MURI on Modular Quantum Circuits. L.E. is also funded by NSF Grant No. DMR-1747426.

\section{APPENDIX A: FREQUENCY DEPENDENCE OF HEATING}

Here we present measurements of the rate of change of the single ion decay parameter $\theta$ as a function of the axial trap frequency $\omega_{0}$. We change the voltages applied to the trap to vary $\omega_{0}$. At each voltage setting, we record carrier Rabi oscillations of the ion after varying wait times $t_{w}$. We fit these data using Eqs. (2)-(3) to extract $d \theta / d t_{w}$. We show the results of these measurements in Fig. 8. We fit the obtained values of $d \theta / d t_{w}$ as a function of the axial frequency $\omega_{0}$ to a power law. To account for the effect of radial heating, we include an offset $B$ into our model by writing $d \theta / d t_{w}=A \omega_{0}^{-2-\alpha}+B$. This gives good agreement of the fit with our data. From the fit, we obtain $\alpha=0.8(1)$, corresponding to electric field noise with power spectral density that scales as $\omega^{-0.8(1)}$. We obtain $B=0.9(1) / \mathrm{s}$, 


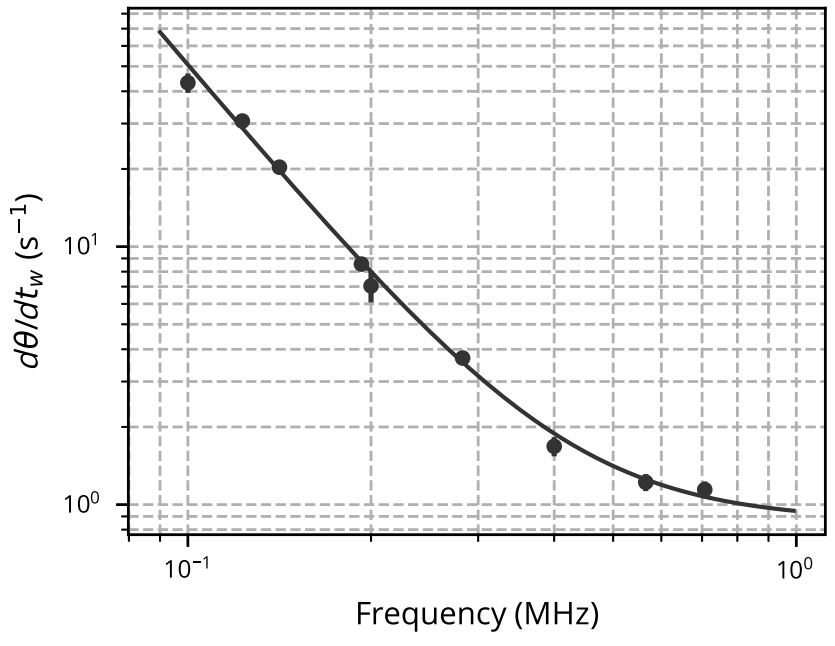

FIG. 8. The rate of change of the decay parameter $\theta$ as a function of the axial trap frequency $\omega_{0}$. The error bars correspond to the $1 \sigma$ fit estimates.

corresponding to $\dot{\bar{n}}_{r}=150(20) / \mathrm{s}$, which agrees with the experimentally measured radial heating for modes oriented at $45^{\circ}$ to the trap surface.

We note that the mean rate of increase in the decay parameter for 15 (25) ions is 1.2 (1.35) times larger than the prediction of Eq. (4). We attribute this to heating of additional modes of motion (not just the in-phase $m=0$ mode), driven by the inhomogeneity of the heating electric field arising from the $70-\mu \mathrm{m}$ distant trap surface across the 62- (106-) $\mu \mathrm{m}$-long ion chain.

\section{APPENDIX B: LOWEST AXIAL FREQUENCY IN EQUISPACED ION CHAINS}

In the limit of many ions $(N \rightarrow \infty)$, an equispaced ion chain can be approximated by a continuous charge distribution with linear charge density $e / d$, where $d$ is the ion spacing. The Coulomb potential of this charge is exactly countered by the applied trap potential

$$
V(x)=\frac{e^{2}}{4 \pi \epsilon_{0} d} \ln \frac{(N / 2)^{2}}{(N / 2)^{2}-(x / d)^{2}},
$$

which holds the ions in place. To model near-equispaced chains, we use $V(x)$ as the trap potential and numerically find the equilibrium ion positions $x_{i, 0}$. We calculate that, when using the potential from Eq. (B1), for all $N<250$, the deviation of the equilibrium ion positions from that of an equal-spaced chain is at most $0.02 d$.

For ion positions $x_{i}$ near the equilibrium, the total energy can be written as $\left(e^{2} / 2 d^{3}\right) \sum_{i, j=1}^{N}\left(x_{i}-x_{i, 0}\right) Q_{i, j}\left(x_{j}-x_{j, 0}\right)$,

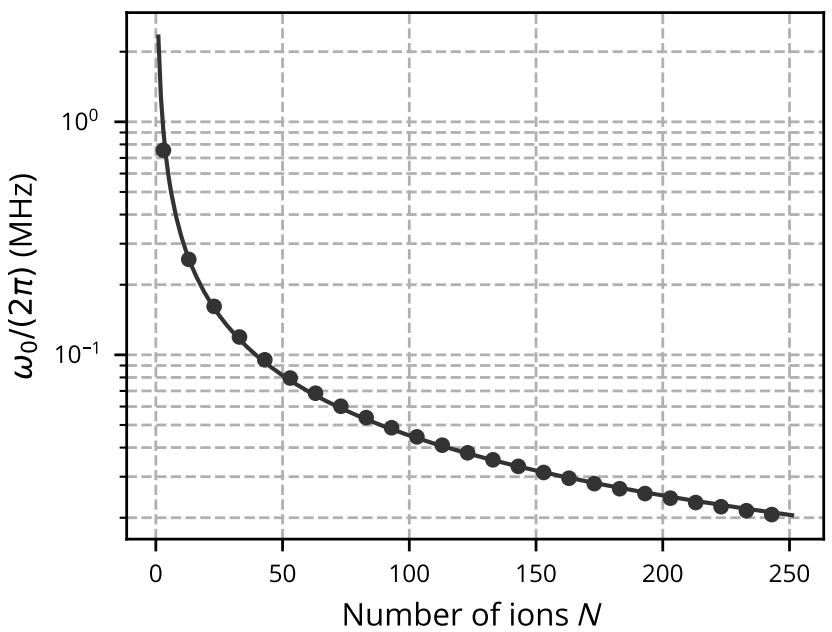

FIG. 9. The lowest axial frequency $\omega_{0}$ of a chain of ${ }^{171} \mathrm{Yb}^{+}$ ions as a function of the number of ions $N$ in the potential given by Eq. (B1) with $d=4.4 \mu \mathrm{m}$. The solid line corresponds to the fitted $N^{-0.856}$ power law.

where

$$
\begin{aligned}
Q_{i, i} & =\frac{2(N / 2)^{2}+2\left(x_{i, 0} / d\right)^{2}}{\left[(N / 2)^{2}-\left(x_{i, 0} / d\right)^{2}\right]^{2}}+\sum_{j \neq i} \frac{2 d^{3}}{\left|x_{i, 0}-x_{j, 0}\right|^{3}} \\
Q_{i \neq j} & =-\frac{2 d^{3}}{\left|x_{i, 0}-x_{j, 0}\right|^{3}} .
\end{aligned}
$$

The axial mode frequencies are then found as $\omega_{m}=$ $\omega_{u} \sqrt{\lambda_{m}}$, where the $\lambda_{m}$ are the eigenvalues of the matrix $Q_{i, j}$ and $\omega_{u}=\sqrt{e^{2} /\left(4 \pi \epsilon_{0} M d^{3}\right)}$ is the frequency scale. The calculated frequency $\omega_{0}$ of the lowest-frequency axial mode is plotted as a function of the ion number, $N$, in Fig. 9. For chains of up to 250 ions, we observe good agreement with the $N^{-0.86}$ scaling that was predicted for chains in harmonic traps in Ref. [29].

\section{APPENDIX C: STATE PREPARATION AND MEASUREMENT ERRORS}

We characterize state preparation and measurement (SPAM) errors for two-qubit gates in a chain of 15 ions by measuring the states of the ions $i=-6,-5$ (see Fig. 3 in the main text) following different state preparations. The qubits are prepared in the states $|00\rangle,|01\rangle,|10\rangle$, and $|11\rangle$, with $2 \times 10^{4}$ trials in each state, and measured in the same basis. We initialize qubits to state $|0\rangle$ using optical pumping; to prepare a qubit in state $|1\rangle$, we rotate the initialized qubit by $\pi$ using a SK1 pulse [37]. To measure the qubit state, we shine $369-\mathrm{nm}$ light, which is resonant with the transition between the $\left\{{ }^{2} S_{1 / 2}, F=1\right\}-\left\{{ }^{2} P_{1 / 2}, F=0\right\}$ manifolds. Ions scatter photons when they are in the $|1\rangle$ (bright) state but not in the $|0\rangle$ (dark) state. Using individual photomultiplier tubes to detect scattered photons from each ion 
TABLE I. State preparation and measurement populations computed on ions $i=-6$ and $j=-5$ (see Fig. 3 in the main text), where states are given by $\left|\psi_{i} \psi_{j}\right\rangle$. The errors correspond to $95 \%$ binomial proportion confidence intervals.

\begin{tabular}{ccccc}
\hline \hline & \multicolumn{4}{c}{ Measured state } \\
\cline { 2 - 5 } Prepared \\
state & $|00\rangle$ & $|01\rangle$ & $|10\rangle$ & $|11\rangle$ \\
\hline$|00\rangle$ & $99.76(7) \%$ & $0.17(6) \%$ & $0.07(4) \%$ & $0.00(2) \%$ \\
$|01\rangle$ & $0.53(10) \%$ & $99.34(12) \%$ & $0.00(2) \%$ & $0.14(5) \%$ \\
$|10\rangle$ & $0.36(9) \%$ & $0.00(2) \%$ & $99.22(12) \%$ & $0.42(9) \%$ \\
$|11\rangle$ & $0.02(2) \%$ & $0.45(10) \%$ & $0.48(10) \%$ & $99.05(14) \%$ \\
\hline \hline
\end{tabular}

for $100 \mu \mathrm{s}$, we determine that the ion is bright (dark) when a measurement generates $>1(\leq 1)$ photons.

SPAM errors are recorded in Table I and used to correct the predicted two-qubit gate fidelities in Fig. 4 of the main text. We emphasize that the measured two-qubit gate fidelities shown in Fig. 4 are not corrected for SPAM errors.

\section{APPENDIX D: COOLING OF RADIAL MODES}

During sympathetic cooling using 435-nm light, we expect the scattered light to recoil-heat off-resonant modes. To counter this, 435-nm light can also be used to cool radial modes. In this appendix we demonstrate such cooling of the 3-MHz antisymmetric radial mode of a pair of one ${ }^{171} \mathrm{Yb}^{+}$and one ${ }^{172} \mathrm{Yb}^{+}$ion.

In Fig. 10 we compare 355-nm Raman-driven Rabi oscillations of the ${ }^{171} \mathrm{Yb}^{+}$qubit after Doppler cooling and following an additional $1 \mathrm{~ms}$ of sympathetic cooling with the two tones of the $435-\mathrm{nm}$ laser tuned $3 \mathrm{MHz}$ to the red of the corresponding $\Delta m=0$ Zeeman lines in

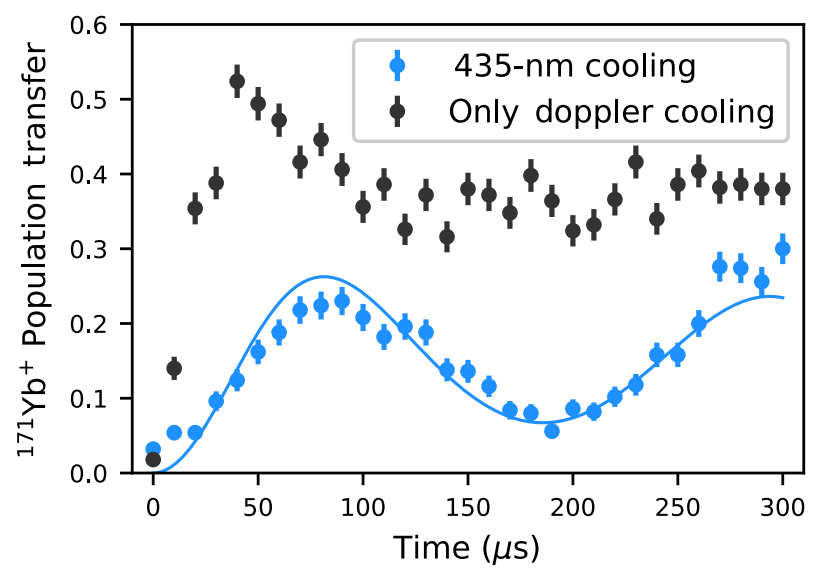

FIG. 10. Raman Rabi oscillations on the red sideband of the antisymmetric radial mode of an ${ }^{171} \mathrm{Yb}^{+}{ }_{-}^{172} \mathrm{Yb}^{+}$ion pair after Doppler cooling (black) and after an additional $1 \mathrm{~ms}$ of 435-nm sympathetic cooling (blue). The fitted blue line corresponds to $\bar{n}=0.42(2)$ residual motional quanta.
${ }^{172} \mathrm{Yb}^{+}$. The rapid cooling of the addressed radial mode to below 0.5 motional quanta demonstrates the value of using near-equal-mass ions for sympathetic cooling.

\section{APPENDIX E: CROSSTALK DURING SYMPATHETIC COOLING}

In this appendix, we calculate theoretical limits on the crosstalk to ${ }^{171} \mathrm{Yb}^{+}$in our cooling scheme.

The strongest decoherence arises from Raman and Rayleigh scattering of 435-nm photons on the D1 and D2 transitions. Following Ref. [38] we determine the damping rate of the ${ }^{171} \mathrm{Yb}^{+}$qubit coherence in our geometry as $\Omega_{435}^{2} \times 1.9 \times 10^{-16} \mathrm{~s}$, where $\Omega_{435}$ is the $435-\mathrm{nm} \mathrm{Rabi}$ frequency on each of the Zeeman transitions in ${ }^{172} \mathrm{Yb}^{+}$during cooling. In order to remain in the resolved-sideband regime and obtain low temperatures, $\Omega_{435}$ needs to be smaller than the motional frequency of the cooled modes. Using the radial mode frequency of $3 \mathrm{MHz}$ from the main text, we find that the resulting qubit decoherence rate is below $7 \times 10^{-2} / \mathrm{s}$, corresponding to an error of at most $3.5 \times 10^{-5}$ per XX gate in our 25-ion chain.

The weaker decoherence, due to scattering on the 435-nm and 297-nm transitions, depends on the relevant isotope shifts. The shifts of the $935-\mathrm{nm}$ transition are available in Ref. [39]. The shifts of the 297-nm transition for even $\mathrm{Yb}$ isotopes were measured by Meyer et al. [40], while the shifts of the 435-nm transition for even isotopes were measured by Counts et al. [41]. A King plot of the modified 435-nm (297-nm) isotope shifts as a function of the modified 935-nm isotope shifts allows us to determine the shift of the centroid of the ${ }^{171} \mathrm{Yb}^{+} 435-\mathrm{nm}$ (297-nm) line relative to ${ }^{172} \mathrm{Yb}^{+}$as $\Delta_{435}=2 \pi \times 1.4 \mathrm{GHz}$ $\left(\Delta_{297}=-2 \pi \times 1.0 \mathrm{GHz}\right)$. Using these shifts, we determine the detunings of the 435-nm, 935-nm, and 297-nm light that is involved in cooling ${ }^{172} \mathrm{Yb}^{+}$from the transitions in ${ }^{171} \mathrm{Yb}^{+}$, and show these in Fig. 11.

The decoherence rate due to scattering on the $435-\mathrm{nm}$ transition is bounded from above by $\Omega_{435}^{2} /\left[4\left(\Delta_{435}^{1 \rightarrow 2}\right)^{2} \tau_{D}\right]$, where $\tau_{D}=52.7 \mathrm{~ms}$ is the lifetime of the ${ }^{2} D_{3 / 2}$ state [30]. For $\Omega_{435}<2 \pi \times 3 \mathrm{MHz}$, this rate is less than $2 \times$ $10^{-5} \mathrm{~s}^{-1}$. Decoherence is also induced by the scattering, by ${ }^{171} \mathrm{Yb}^{+}$, of $297-\mathrm{nm}$ photons via the off-resonant, two-step, $F=1 \rightarrow F=1 \rightarrow F=0$ transition that is driven by the combination of the 435-nm and the 935-nm light used to cool ${ }^{172} \mathrm{Yb}^{+}$. We model this process using three-level optical Bloch equations. Assuming 435-nm and 935-nm Rabi frequencies of $2 \pi \times 3 \mathrm{MHz}$, we find that the rate of scattering of $297-\mathrm{nm}$ photons by a ${ }^{171} \mathrm{Yb}^{+}$ion via this process, and the resulting decoherence rate, are both smaller than $2 \times 10^{-5} \mathrm{~s}^{-1}$.

Crosstalk arises due to absorption, by ${ }^{171} \mathrm{Yb}^{+}$, of the 297-nm photons that are scattered by ${ }^{172} \mathrm{Yb}^{+}$. Given the near-unity branching ratio of the $297-\mathrm{nm}$ transition, a single ${ }^{172} \mathrm{Yb}^{+}$ion at unity saturation will scatter $297-\mathrm{nm}$ 


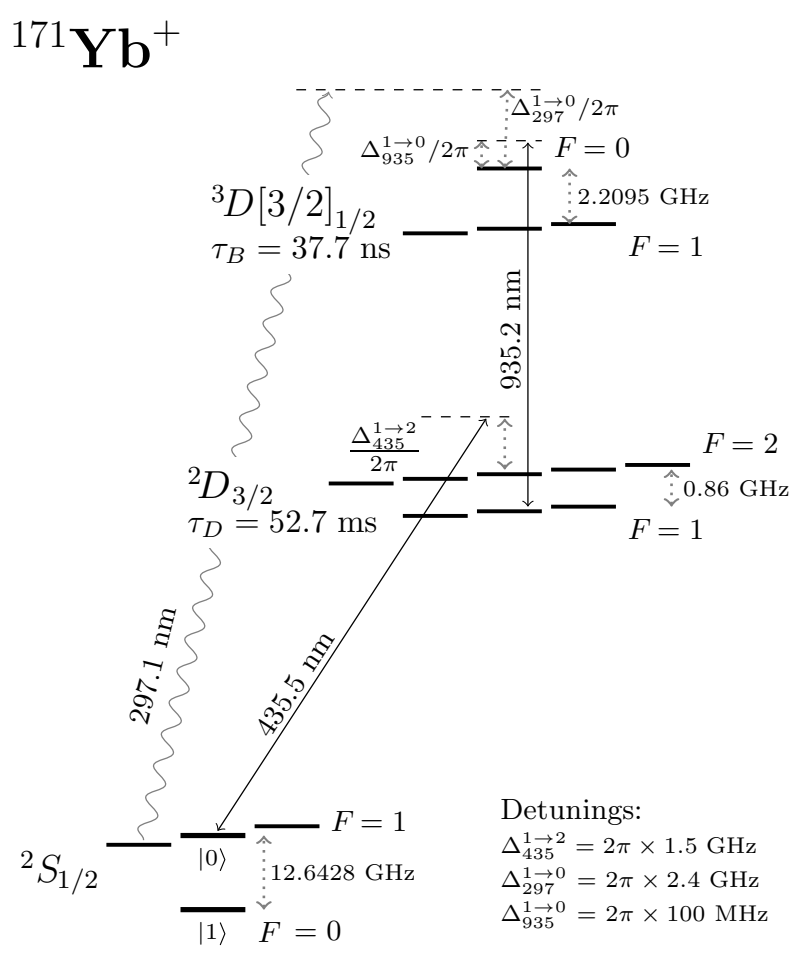

FIG. 11. Diagram of the states and transitions involved in (a) the proposed cooling scheme of ${ }^{172} \mathrm{Yb}^{+}$and (b) the crosstalk to the ${ }^{171} \mathrm{Yb}^{+}$qubit ions. Lifetime of the ${ }^{2} D_{3 / 2}$ state is cited from Ref. [30], and that of the ${ }^{3} D[3 / 2]_{1 / 2}$ state is from Ref. [31]. The hyperfine splittings of the ${ }^{3} D[3 / 2]_{1 / 2}$ and ${ }^{2} D_{3 / 2}$ states are from Ref. [32].

photons at the rate $\Gamma_{\mathrm{sc}}=\Gamma / 4$, where $\Gamma=1 / \tau_{B}$, with $\tau_{B}=$ $37.7 \mathrm{~ns}$ the lifetime of the ${ }^{3} D[3 / 2]_{1 / 2}$ state [31]. These scattered photons will be absorbed by $\mathrm{a}^{171} \mathrm{Yb}^{+}$ion at distance $d$ at the maximal rate

$$
\Gamma_{297}^{\max }=6 \pi\left(\frac{\lambda}{2 \pi}\right)^{2} \frac{\Gamma / 4}{4 \pi d^{2}}\left(\frac{\Gamma / 2}{\Delta_{1 \rightarrow 0}^{297}}\right)^{2},
$$

where $\lambda=297 \mathrm{~nm}$. Considering a single ${ }^{171} \mathrm{Yb}^{+}$ion in an infinite chain with spacing $d$ and fraction $r$ of ${ }^{172} \mathrm{Yb}^{+}$ions yields the expression for the scattering rate $R$ in the main text with $\Delta=\Delta_{297}^{\max }$. Setting $d=4 \mu \mathrm{m}$ and $r=0.5$ then yields $R<2 \times 10^{-3} \mathrm{~s}^{-1}$.

[1] R. Blatt and D. Wineland, Entangled states of trapped atomic ions, Nature 453, 1008 (2008).

[2] C. Monroe and J. Kim, Scaling the ion trap quantum Processor, Science 339, 1164 (2013).

[3] C. J. Ballance, T. P. Harty, N. M. Linke, M. A. Sepiol, and D. M. Lucas, High-Fidelity Quantum Logic Gates Using Trapped-Ion Hyperfine Qubits, Phys. Rev. Lett. 117, 060504 (2016).
[4] J. P. Gaebler, T. R. Tan, Y. Lin, Y. Wan, R. Bowler, A. C. Keith, S. Glancy, K. Coakley, E. Knill, D. Leibfried, and D. J. Wineland, High-Fidelity Universal Gate Set for ${ }^{9} \mathrm{Be}^{+}$Ion Qubits, Phys. Rev. Lett. 117, 060505 (2016).

[5] D. Kielpinski, C. Monroe, and D. J. Wineland, Architecture for a large-scale ion-trap quantum computer, Nature 417, 709 (2002).

[6] J. M. Pino, J. M. Dreiling, C. Figgatt, J. P. Gaebler, S. A. Moses, M. S. Allman, C. H. Baldwin, M. Foss-Feig, D. Hayes, K. Mayer, C. Ryan-Anderson, and B. Neyenhuis, Demonstration of a small programmable quantum computer with atomic qubits, Nature 563, 63 (2016).

[7] C. Monroe, R. Raussendorf, A. Ruthven, K. R. Brown, P. Maunz, L.-M. Duan, and J. Kim, Large-scale modular quantum-computer architecture with atomic memory and photonic interconnects, Phys. Rev. A 89, 022317 (2014).

[8] K. Mølmer and A. Sørensen, Multiparticle Entanglement of Hot Trapped Ions, Phys. Rev. Lett. 82, 1835 (1999).

[9] G. J. Milburn, S. Schneider, and D. F. V. James, Ion trap quantum computing with warm ions, Fort. Physik 48, 801 (2000).

[10] E. Solano, R. L. de Matos Filho, and N. Zagury, Deterministic bell states and measurement of the motional state of two trapped ions, Phys. Rev. A 59, R2539 (1999).

[11] S.-L. Zhu, C. Monroe, and L.-M. Duan, Trapped Ion Quantum Computation with Transverse Phonon Modes, Phys. Rev. Lett. 97, 050505 (2006).

[12] Q. A. Turchette, D. Kielpinski, B. E. King, D. Leibfried, D. M. Meekhof, C. J. Myatt, M. A. Rowe, C. A. Sackett, C. S. Wood, W. M. Itano, C. Monroe, and D. J. Wineland, Heating of trapped ions from the quantum ground state, Phys. Rev. A 61, 063418 (2000).

[13] M. Brownnutt, M. Kumph, P. Rabl, and R. Blatt, Iontrap measurements of electric-field noise near surfaces, Rev. Mod. Phys. 87, 1419 (2015).

[14] S. Debnath, N. M. Linke, C. Figgatt, K. A. Landsman, K. Wright, and C. Monroe, Demonstration of a small programmable quantum computer with atomic qubits, Nature 563, 63 (2016).

[15] K. A. Landsman, Y. Wu, P. H. Leung, D. Zhu, N. M. Linke, K. R. Brown, L. Duan, and C. Monroe, Two-qubit entangling gates within arbitrarily long chains of trapped ions, Phys. Rev. A 100, 022332 (2019).

[16] K. Wright et al., Benchmarking an 11-qubit quantum computer, Nat. Commun. 10, 5464 (2019).

[17] P. Jurčević, (PhD thesis) University of Innsbruck, 2017.

[18] M. Saffman, Quantum computing with atomic qubits and rydberg interactions: Progress and challenges, J. Phys. B: At. Mol. Opt. Phys. 49, 202001 (2016).

[19] L. Egan, D. M. Debroy, C. Noel, A. Risinger, D. Zhu, D. Biswas, M. Newman, M. Li, K. R. Brown, M. Cetina, and C. Monroe, arXiv:2009.11482 [quant-ph] (2020).

[20] D. J. Larson, J. C. Bergquist, J. J. Bollinger, W. M. Itano, and D. J. Wineland, Sympathetic Cooling of Trapped Ions: A Laser-Cooled Two-Species Nonneutral ion Plasma, Phys. Rev. Lett. 57, 70 (1986).

[21] C. W. Chou, D. B. Hume, J. C. J. Koelemeij, D. J. Wineland, and T. Rosenband, Frequency Comparison of Two HighAccuracy $\mathrm{Al}^{+}$Optical Clocks, Phys. Rev. Lett. 104, 070802 (2010). 
[22] D. F. V. James, Quantum dynamics of cold trapped ions with application to quantum computation, Appl. Phys. B 66, 181 (1998).

[23] A. D. West, R. Putnam, W. Campbell, and P. Hamilton, Tunable transverse spin-motion coupling for quantum information processing, Quantum. Sci. Technol. 6, 024003 (2021).

[24] P. L. W. Maunz, Sandia National Laboratories Report No. SAND2016-0796R (2016).

[25] D. Leibfried, R. Blatt, C. Monroe, and D. Wineland, Quantum dynamics of single trapped ions, Rev. Mod. Phys. 75, 281 (2003).

[26] M. A. Nielsen and I. L. Chuang, Quantum Computation and Quantum Information (Cambridge University Press, Cambridge, UK, 2011).

[27] G.-D. Lin, S.-L. Zhu, R. Islam, K. Kim, M.-S. Chang, S. Korenblit, C. Monroe, and L.-M. Duan, Large-scale quantum computation in an anharmonic linear ion trap, EPL 86, 60004 (2009).

[28] C. A. Sackett, D. Kielpinski, B. E. King, C. Langer, V. Meyer, C. J. Myatt, M. Rowe, Q. A. Turchette, W. M. Itano, and D. J. Wineland, Experimental entanglement of four particles, Nature 404, 256 (2000).

[29] J. P. Schiffer, Phase Transitions in Anisotropically Confined Ionic Crystals, Phys. Rev. Lett. 70, 818 (1993).

[30] N. Yu and L. Maleki, Lifetime measurements of the $4 f^{14}$ $5 d$ metastable states in single ytterbium ions, Phys. Rev. A 61, 022507 (2000).

[31] R. W. Berends, E. H. Pinnington, B. Guo, and Q. Ji, Beam-laser lifetime measurements for four resonance levels of yb II, J. Phys. B: At. Mol. Opt. Phys. 26, L701 (1993).

[32] S. Olmschenk, K. C. Younge, D. L. Moehring, D. N. Matsukevich, P. Maunz, and C. Monroe, Manipulation and detection of a trapped $\mathrm{Yb}^{+}$hyperfine qubit, Phys. Rev. A 76, 052314 (2007).

[33] M. Revelle, C. W. Hogle, B. Ruzic, P. L. W. Maunz, K. Young, and D. Lobser, Sandia National Laboratories Report No. SAND2019-0668C671715 (2019).

[34] C. P. Slichter, Principles of Magnetic Resonance (SpringerVerlag, Berlin, Heidelberg, 1996).

[35] L. Karpa, A. Bylinskii, D. Gangloff, M. Cetina, and V. Vuletić, Suppression of Ion Transport due to LongLived Subwavelength Localization by an Optical Lattice, Phys. Rev. Lett. 111, 163002 (2013).

[36] C. Schneider, M. Enderlein, T. Huber, and T. Schaetz, Optical trapping of an ion, Nat. Photonics 4, 772 (2010).

[37] J. T. Merrill and K. R. Brown, in Quantum Information and Computation for Chemistry (John Wiley \& Sons, Ltd, 2014), p. 241.

[38] H. Uys, M. J. Biercuk, A. P. VanDevender, C. Ospelkaus, D. Meiser, R. Ozeri, and J. J. Bollinger, Decoherence due to Elastic Rayleigh Scattering, Phys. Rev. Lett. 105, 200401 (2010).

[39] J. J. McLoughlin, A. H. Nizamani, J. D. Siverns, R. C. Sterling, M. D. Hughes, B. Lekitsch, B. Stein, S. Weidt, and W. K. Hensinger, Versatile ytterbium ion trap experiment for operation of scalable ion-trap chips with motional heating and transition-frequency measurements, Phys. Rev. A 83, 013406 (2011).

[40] H. M. Meyer, M. Steiner, L. Ratschbacher, C. Zipkes, and M. Köhl, Laser spectroscopy and cooling of $\mathrm{Yb}^{+}$ions on a deep-UV transition, Phys. Rev. A 85, 012502 (2012).

[41] I. Counts, J. Hur, D. P. L. Aude Craik, H. Jeon, C. Leung, J. C. Berengut, A. Geddes, A. Kawasaki, W. Jhe, and V. Vuletić, Evidence for Nonlinear Isotope Shift in $\mathrm{Yb}^{+}$ Search for New Boson, Phys. Rev. Lett. 125, 123002 (2020). 